\title{
Nanoparticles engineered for antigen-specific immunotherapy
}

Current therapeutics for autoimmune conditions are usually nonspecific and induce general immune suppression. Now, reporting in Nature, Clemente-Cesares and colleagues show that nanoparticles coated with autoimmune-disease relevant peptides bound to $\mathrm{MHC}$ class II (pMHC-NPs) can induce antigen-specific $\mathrm{T}$ cells with regulatory function in vivo. This approach led to suppression of disease in mouse models of rheumatoid arthritis (RA), type 1 diabetes (T1D) and multiple sclerosis (MS), without affecting general immunity.

Autoimmune disease results from chronic T cell and B cell responses to autoantigens. Central to this process are autoantigenresponsive $\mathrm{CD}^{+} \mathrm{T}$ helper $\left(\mathrm{T}_{\mathrm{H}}\right)$ cells,

pMHC-NPs
promote the
differentiation
of rare
pre-activated
autoreactive
CD4+ T cells
into TR 1 -like
cells

which orchestrate the function of other immune cells. Conversely, $\mathrm{CD}^{+} \mathrm{T}$ cells with regulatory function ( $\mathrm{T}_{\mathrm{Reg}}$ cells), including $\mathrm{FOXP}^{+}{ }^{+} \mathrm{CD} 4{ }^{+} \mathrm{CD} 25^{+} \mathrm{T}_{\mathrm{Reg}}$ cells and FOXP3-CD $4^{+} \mathrm{CD} 25^{-} \mathrm{T}_{\mathrm{R}} 1$ cells, can dampen the inflammatory process.

Based on the hypothesis that chronic autoantigen exposure may convert autoreactive $\mathrm{T}$ cells into regulatory $\mathrm{T}$ cells in a negative feedback loop, the authors engineered nanoparticles coated with the pMHC complex $2.5 \mathrm{mi} / \mathrm{IA}^{\mathrm{g} 7}$, which is a known antigenic target in the development of T1D in non-obese diabetic (NOD) mice. After intravenous injection with these pMHC-NPs twice weekly for 5 weeks, the animals developed antigen-specific $\mathrm{CD} 4^{+} \mathrm{T}$ cells of the memory-like (CD44 $\left.{ }^{\text {hi }} \mathrm{CD} 62 \mathrm{~L}^{\text {low }}\right)$ FOXP $^{-} \mathrm{T}_{\mathrm{R}} 1$-like phenotype and reverted to stable normoglycaemia. Similar results were achieved when the nanoparticles were coated with pMHC complexes that contained sub-dominant (non-disease causing) NOD-specific autoantigenic peptides - but uncoated nanoparticles, pMHC monomers, peptides, peptide-coated nanoparticles or peptide-coated microparticles had no effect.

A similar strategy was used to reverse paralysis in mice with autoimmune encephalomyelitis (EAE; a model of MS) and to reduce joint inflammation in collagen-induced arthritis (CIA; a model of RA). The effect was strictly dependent on the presence of the disease-specific autoantigen-MHC complex - that is, the nanoparticles that were effective in the EAE model had no effect in the CIA model, and vice versa. However, disease-specific autoantigens besides the ones used to induce disease had therapeutic activity, indicating that the therapeutic effects of pMHC-NPs are disease-specific but dissociated from the pathogenic role of the antigenic epitope that is presented.

Further in vitro and adoptive transfer experiments indicated that pMHC-NPs promote the differentiation of rare pre-activated autoreactive $\mathrm{CD}^{+} \mathrm{T}$ cells into $\mathrm{T}_{\mathrm{R}} 1$-like cells, followed by their systemic expansion. The $\mathrm{T}_{\mathrm{R}}$ 1-like cells suppressed autoreactive helper and cytotoxic T cells through the secretion of interleukin-10 (IL-10), transforming growth factor- $\beta$ (TGF $\beta$ ) and IL- 21 . Moreover, $\mathrm{T}_{\mathrm{R}} 1$-like cells induced the formation and expansion of regulatory $\mathrm{B}$ cells and downregulated the production of pro-inflammatory mediators by antigen-presenting cells. Importantly, this did not impair general immunity, as pMHC-NP-treated mice cleared acute viral infections as efficiently as untreated mice. The potential translatability of the approach to human disease was demonstrated by using human T1D-relevant pMHC-NPs in NOD severe combined immunodeficient $\mathrm{Il}_{2 \mathrm{rg}^{-1-}}$ mice reconstituted with peripheral blood mononuclear cells from T1D patients.

The authors suggest that pMHCNPs may form a new class of therapeutics capable of resolving complex autoimmune disease in a diseaseand organ-specific manner, without compromising systemic immunity.

Alexandra Flemming 